\title{
Técnica del amarre iliofemoral utilizando la sutura de anclaje (anchor suture) sobre la corrección de las luxaciones coxofemorales craneodorsales en perros (Canis familiaris)
}

\author{
Technique of the Iliofemoral Attachment Using the Anchor Suture over the \\ Correction of Coxofemoral Craniodorsal Luxations in Dog (Canis familiaris)
}

\author{
Lorena B. Silva Roeder ${ }^{*}$, Lyana Quispe Ochoa ${ }^{* *}$, Maite Baquerizo Revilla ${ }^{* *}$
}

http://dx.doi.org/10.21503/CienciayDesarrollo.2011.v14.03

\section{RESUMEN}

El objetivo de esta investigación ha sido demostrar que el uso de la técnica del amarre iliofemoral utilizando la sutura de anclaje corrige las luxaciones coxofemorales craneodorsales en perros (Canis familiaris). La recolección de datos y la realización de esta investigación se iniciaron en el mes de marzo y finalizó en el mes de agosto del 2010. La muestra constó de 7 caninos urbano-hogareños que fueron diagnosticados radiológicamente con luxación coxofemoral craneodorsal. De acuerdo con los registros de estado clínico prequirúrgico y posquirúrgico, se encontró que el $100 \%$ de los pacientes se recuperó completamente en un tiempo menor a los 30 días posteriores a la cirugía. Se concluye, entonces, que el uso de la técnica del amarre iliofemoral utilizando la sutura de anclaje corrige las luxaciones coxofemorales craneodorsales en perros (Canisfamiliaris).

Palabras clave: sutura, iliotrocantérica, anclaje, luxación, cadera.

\section{ABSTRACT}

The aim of this research was to demonstrate that the use of the technique of the iliofemoral tie using the anchor suture corrects craniodorsal coxofemoral luxations in dogs (Canisfamiliaris). Data collection and conduct of this investigation was initiated in March and ended in August 2010. The sample consisted of 7 urban-household dogs that were diagnosed radiologically with craniodorsal hip dislocation. According to the records of pre and post operative clinical status was found that $100 \%$ of the patients recovered completely in a time less than 30 days after surgery. It is concluded that the use of the technique of the iliofemoral tie using the anchor suture corrects craniodorsal coxofemoral luxation in dogs (Canis familiaris).

Key words: suture, iliotrocanteric, anchoring, dislocation, hip.

* Médica veterinaria graduada en la EAP de Medicina Veterinaria de la UAP.

** Docente de la UAP. EAP de Medicina Veterinaria.

*** Docente de la UAP. EAP de Medicina Veterinaria. (maitebaquerizo@yahoo.es) 


\section{INTRODUCCIÓN}

La ortopedia canina va cobrando importancia porque los propietarios muestran un creciente interés por corregir las lesiones traumatológicas de sus mascotas.

Existen numerosas técnicas para corregir las luxaciones coxofemorales craneodorsales en perros $(1,4)$. La más utilizada es la sutura estabilizadora iliofemoral o amarre iliofemoral; sin embargo, el inconveniente de esta técnica es que la ligadura resulta muy laboriosa y riesgosa debido a que por debajo del lugar donde se realiza el agujero recorre un paquete neurovascular importante y el período de recuperación es extenso(4). Se describe que la técnica del amarre iliofemoral utilizando la sutura de anclaje resulta $10 \%$ más costosa que la tradicional, pero al reducir el traumatismo muscular se reduce también el tiempo de recuperación(5). El uso de esta técnica minimiza el traumatismo de los músculos glúteos al no manipularlos en exceso para hacer la ligadura, lo cual permite una ambulación postquirúrgica inmediata.

El objetivo del presente trabajo de investigación fue evaluar cómo la técnica del amarre iliofemoral utilizando la sutura de anclaje tiene un efecto correctivo sobre las luxaciones coxofemorales craneodorsales en perros (Canis familiaris).

\section{MATERIALES Y MÉTODOS}

Los individuos utilizados para la investigación fueron pacientes de diferentes clínicas veterinarias de la ciudad de Lima, los cuales fueron derivados a la clínica ubicada en Miraflores. Los casos de luxaciones coxofemorales craneodorsales corresponden a un total de 7 individuos con edades de 1 año 2 meses a 7 años 5 meses. Los caninos fueron sometidos a un examen prequirúrgico.
Después de inducido el paciente en la cirugía, se procedió a palpar y localizar la tuberosidad isquiática, el trocánter mayor del fémur y el ala del ilion. La incisión se realizó sobre el mismo fémur con dirección craneal. Se identificó el músculo tensor de fascia lata y bíceps femoral. Se incidió la hoja profunda de fascia lata en dirección distal hacia proximal, redirigiendo el corte hacia craneal.

Con un retractor Ghelpi se desplazó a caudal el músculo bíceps femoral, se ubicó el músculo glúteo superficial y medio debajo del músculo tensor de la fascia lata y se reflejó hacia proximal, donde apareció el músculo glúteo profundo. Debajo de este músculo se palpó el gran trocánter y el cuello femoral.

Ubicados el acetábulo, el trocánter mayor y la cabeza femoral, se procedió a realizar una perforación en el borde craneal del acetábulo, empleando una broca de 3,2 $\mathrm{mm}$ para colocar en este agujero un anclaje de sutura (anchor suture). Luego se realizó un túnel por debajo del trocánter mayor, por el cual se insertó un extremo de hilo de material no absorbible (nylon) que fue amarrado al otro extremo del mismo insertado por el agujero del seguro. Ya insertados ambos extremos del hilo, se procedió a realizar un amarre con nudo cuadrado, el cual aseguró la articulación y previno una reluxación. Finalmente, se reconstruyó y cerró la cápsula articular y las estructuras, que fueron incididas.

El tratamiento farmacológico posquirúrgico constó de aplicación de enrofloxacina, carprofeno y tramadol. Los pacientes permanecieron en confinamiento moderado durante un tiempo mínimo de 2 semanas.

Diez días después de la cirugía, se retiraron las suturas externas y se realizó un nuevo examen clínico que constó de examen en dinámica y examen físico. A los 30 días postcirugía se realizó el mismo procedimiento, incluyendo examen radiológico de control. 
Los datos registrados durante el examen clínico posquirúrgico fueron los siguientes:

a) Grado de claudicación: I = Apenas perceptible, II = Notable pero apoya el miembro afectado, III = Apoya el miembro afectado solo para equilibrarse, y IV $=$ No apoya y mantiene el miembro en flexión (6).

b) Dolor del arco de movilidad: mediante la escala de Melbourne (7), donde los datos requeridos son la frecuencia cardiaca, frecuencia respiratoria, temperatura rectal, midriasis y salivación.

Cada descriptor tiene un valor que indica el grado de dolor: $0=$ Sin dolor, $1=$ Dolor leve, $2=$ Dolor moderado, $3=$ Dolor severo. Los valores obtenidos en cada variable se sumaron y se obtuvo el valor total de la escala para cada paciente. El valor mínimo posible de la sumatoria de la escala es el 0 , y el máximo, 12 .

El valor igual a cero $(0)$ indica ausencia de dolor, entre 1 y 4 indica dolor leve, entre 5 y 8 indica dolor moderado, y entre 9 y 12 , dolor severo.

c) Simetría de los miembros: se midió en $\mathrm{cm}$ mediante la observación de ambos miembros estirados.

d) Congruencia articular:medianteobservación de la radiografía control.
Se procedió a realizar la comparación entre el estado prequirúrgico y posquirúrgico del paciente.

El efecto de la técnica se midió según el porcentaje de individuos que recuperaron totalmente la actividad del miembro afectado, siendo esto resultado de la disminución de los indicadores al cabo de 30 días de realizada la intervención quirúrgica.

\section{RESULTADOS}

\section{Recuperación del paciente según grado de claudicación}

En la tabla 1 se puede observar un paciente con grado II de claudicación, equivalente a 14,3 \%; 4 pacientes con grado III de claudicación, equivalentes a $57,1 \%$, y 2 pacientes con grado IV de claudicación, que equivalen a 28,6 \%. Diez días después de realizada la cirugía, un paciente presentaba grado 0 de claudicación, que equivale a $14,3 \%$; 4 pacientes presentaban grado I de claudicación, que equivalen a $57,1 \%$, y 2 pacientes presentaban grado II de claudicación, que equivalen a 28,6 \%. Treinta días después de la realización de la cirugía, los 7 pacientes presentaron grado 0 de claudicación, es decir, el total de ellos se había recuperado.

\section{Tabla 1. Grado de claudicación}

\begin{tabular}{c|c|c|c|c|c|c|}
\multirow{2}{*}{$\begin{array}{c}\text { Grado de } \\
\text { claudicación }\end{array}$} & \multicolumn{2}{|c|}{ Día 0 } & \multicolumn{2}{c|}{ Día 10 } & \multicolumn{2}{c|}{ Día 30} \\
\cline { 2 - 7 } & $\mathrm{n}$ & $\%$ & $\mathrm{n}$ & $\%$ & $\mathrm{n}$ & $\%$ \\
\hline I & - & - & 1 & 14,3 & 7 & 100 \\
\hline II & - & - & 4 & 57,1 & - & - \\
III & 1 & 14,3 & 2 & 28,6 & - & - \\
IV & 4 & 57,1 & - & - & - & - \\
\hline
\end{tabular}


Tabla 2. Dolor a evaluación del arco de movilidad

\begin{tabular}{l|c|c|c|c|c|c|}
\multirow{2}{*}{$\begin{array}{l}\text { Dolor al arco } \\
\text { de movilidad }\end{array}$} & \multicolumn{2}{|c|}{ Día 0 } & \multicolumn{2}{c|}{ Día 10 } & \multicolumn{2}{c}{ Día 30 } \\
\cline { 2 - 8 } & $\mathrm{n}$ & $\%$ & $\mathrm{n}$ & $\%$ & $\mathrm{n}$ & $\%$ \\
\hline Ninguno & 0 & - & 0 & - & 7 & 100 \\
\hline Leve & 1 & 14,3 & 5 & 71,4 & 0 & - \\
\hline Moderado & 3 & 42,9 & 2 & 28,6 & 0 & - \\
\hline Severo & 3 & 42,9 & 0 & - & 0 & - \\
\hline
\end{tabular}

Recuperación del paciente según dolor al arco de movilidad

En la tabla 2 se observa que un paciente presentaba dolor leve a la evaluación del arco de movilidad, que equivale a $14,3 \% ; 3$ presentaban dolor moderado a la evaluación del arco de movilidad, equivalentes al 42,9\%, y 3 presentaban dolor severo a la evaluación del arco de movilidad, que equivalen a $42,9 \%$. 10 días después de la realización de la cirugía, 5 pacientes presentaban dolor leve a la evaluación del arco de movilidad, que equivalen a 71,4\%; 2 presentaban dolor moderado a la evaluación del arco de movilidad, que equivalen a $28,6 \%$. 30 días después de la realización de la cirugía, 7 pacientes no presentaban ningún dolor a la evaluación del arco de movilidad, que equivalen al $100 \%$ de los pacientes.
Recuperación del paciente según asimetría de los miembros

En la tabla 3, según los indicadores, se observa que antes de la realización de la cirugía, 2 pacientes presentaban $2 \mathrm{~cm}$ de asimetría de los miembros, que equivalen a $28,6 \%$; un paciente presentaba $2,2 \mathrm{~cm}$ de asimetría de los miembros, que equivale a $14,3 \%$; un paciente presentaba 2,5 $\mathrm{cm}$ de asimetría de los miembros, que equivale a $14,3 \%$; un paciente presentaba $3 \mathrm{~cm}$ de asimetría de los miembros, que equivale a $1,3 \%$; un paciente presentaba $3,2 \mathrm{~cm}$ de asimetría de los miembros, que equivale a $1,3 \%$, y un paciente presentaba 4 $\mathrm{cm}$ de asimetría de los miembros, que equivale a $14,3 \%$. Diez días después de la realización de la cirugía, 7 pacientes no presentaban ninguna asimetría de los miembros, que equivalen a $100 \%$, y 30 días después de la realización de la cirugía, 7 pacientes no presentaban ninguna asimetría de los miembros, que equivalen a $100 \%$.

Tabla 3. Asimetría de los miembros

\begin{tabular}{c|c|c|c|c|c|c|}
\hline \multirow{2}{*}{$\begin{array}{c}\text { Asimetría de los } \\
\text { miembros } \\
(\mathrm{cm})\end{array}$} & \multicolumn{2}{|c|}{ Día 0 } & \multicolumn{2}{c|}{ Día 10 } & \multicolumn{2}{c|}{ Día 30 } \\
\cline { 2 - 8 } $0 \mathrm{~cm}$ & $\mathrm{n}$ & $\%$ & $\mathrm{n}$ & $\%$ & $\mathrm{n}$ & $\%$ \\
\hline $2 \mathrm{~cm}$ & 0 & - & 7 & 100 & 7 & 100 \\
\hline $2,2 \mathrm{~cm}$ & 2 & 28,6 & 0 & - & 0 & - \\
\hline $2,5 \mathrm{~cm}$ & 1 & 14,3 & 0 & - & 0 & - \\
\hline $3,0 \mathrm{~cm}$ & 1 & 14,3 & 0 & - & 0 & - \\
\hline $3,2 \mathrm{~cm}$ & 1 & 14,3 & 0 & - & 0 & - \\
$4,0 \mathrm{~cm}$ & 1 & 14,3 & 0 & - & 0 & - \\
\hline
\end{tabular}


Recuperación del paciente según congruencia articular

En tabla 4 se observa, según los indicadores, que antes de la realización de la cirugía, 7 pacientes no presentaban congruencia articular, que equivalen a 100\%. Diez días después de la realización de la cirugía, 7 pacientes sí presentaban congruencia articular, que equivale, a 100\%, y 30 días después de la realización de la cirugía, 7 pacientes sí presentaban congruencia articular, que equivalen a $100 \%$.

\section{Tabla 4. Congruencia articular}

\begin{tabular}{c|c|c|c|c|c|c}
\multirow{2}{*}{$\begin{array}{c}\text { Congruencia } \\
\text { articular }\end{array}$} & \multicolumn{6}{|c|}{ Día de observación } \\
\cline { 2 - 8 } & \multicolumn{2}{|c|}{ Día 0 } & \multicolumn{2}{|c|}{ Día 10 } & \multicolumn{3}{|c}{ Día 30 } \\
\cline { 2 - 7 } & $\mathrm{n}$ & $\%$ & $\mathrm{n}$ & $\%$ & $\mathrm{n}$ & $\%$ \\
\hline Sí & 0 & - & 7 & 100 & 7 & 100 \\
\hline No & 7 & 100 & 0 & - & 0 & - \\
\hline
\end{tabular}

\section{DISCUSIÓN}

Antes de la cirugía, todos los pacientes presentaban grados de claudicación del grado II al grado IV, producto de la luxación. Diez días después de la intervención, un paciente presentaba grado 0 de claudicación, 4 pacientes grado 1, y 2 pacientes grado II, esto debido al dolor producido por la manipulación de la articulación en la cirugía y a la debilidad de la fibrosis pericapsular primaria. Treinta días después, los 7 pacientes presentaban grado de claudicación 0 , ya que la fibrosis que estabiliza la articulación se había instaurado, recuperándose la biomecánica articular normal de la cadera, coincidiendo con lo manifestado por Mucha (4) y Reyes (8).

Respecto al dolor al arco de movilidad, antes de la cirugía los pacientes presentaban dolor leve, moderado o severo, producto de la alteración de la superficie de la cabeza femoral debida a su contacto inadecuado con el ilion, según lo descrito por Morales en el 2004 (1). Diez días después del tratamiento, 5 pacientes presentaban dolor leve, y 2, dolor moderado, debido a la manipulación y sección de estructuras en la cirugía. Treinta días después, los 7 pacientes no presentaban ningún dolor, ya que tanto la integridad así como la anatomía normal de la cadera habían sido recuperadas.

En cuanto a la asimetría, antes del tratamiento, los pacientes presentaban asimetría de los miembros que variaba de los $2 \mathrm{~cm}$ hasta los $4 \mathrm{~cm}$, coincidiendo con lo señalado por Tabar (2) y Santoscoy (6). Diez días después de la cirugía, los 7 pacientes no presentaban asimetría en los miembros, y lo mismo se observó a los 30 días, puesto que la disposición normal de los componentes de la articulación había sido recobrada.

\section{CONCLUSIÓN}

Los resultados de este trabajo experimental nos permiten concluir que la técnica de la sutura de anclaje corrigió las luxaciones coxofemorales craneodorsales de los 7 casos presentados, con lo que a los 30 días posteriores a la cirugía se produjo la recuperación total de la actividad del miembro afectado en el $100 \%$ de los pacientes.

\section{REFERENCIAS BIBLIOGRÁFICAS}

1. Morales J.L. "Anatomía aplicada de los pequeños animales". En: http://www.uco. es/organiza/departamentos/anatomia-yanat-patologica/peques/luxacadera 2004. pdf. Acceso el 10 abril de 2009.

2. Tabar J.J.I. "Mis técnicas favoritas en la luxación de cadera”. En: http://www.avepa. org/grupos/gevo/jornadas01/art03.htm. Acceso el 10 abril de 2009. 
3. Sánchez Valverde M. Traumatología y ortopedia de pequeños animales. México, Mc Graw-Hill-Interamericana, 1998.

4. Mucha C. Consulta rápida en la clínica diaria. Argentina, Inter-Médica, 2005.

5. Fossum T. Cirugia en pequeños animales. $3^{\mathrm{a}}$ ed. Argentina, Inter-Medica, 1999.

6. Santoscoy C. "Examen diagnóstico en ortopedia”. En: http://www.ammvepe.com/ articulos/ortopedia.html. Acceso el 14 de mayo de 2009.
7. Universidad de Zaragoza. "Comisión ética asesora para la experimentación animal". En: http://cea.unizar.es/Disenos experimentales/Anestesia $\% 20$ y 20 analgesia/Evaluacion_dolor/Evaluacion_ dolor_en_perro.pdf_. Acceso el 18 mayo de 2010.

8. Reyes J.H. "Articulación coxofemoral del perro doméstico". En: http://www. ammvepen.com.mx/Pdf/DISPLASIA\%20 DE\%20CADERA.pdf. Acceso el 30 de mayo de 2009. 